\section{(0) Vorrex}

IRIT - University of Toulouse - France

\section{Monte-Carlo rendering}

Value of each pixel defined as the expected value of a random variable $X$ $I=E[X]$

- estimated using samples of $X$

$$
<I>=\frac{1}{N} \sum_{i=1}^{N} x_{i}
$$

\section{Our goals}

- Focus processing power where convergence is harder to reach during Monte-Carlo based rendering

- Make the error over the pixels uniform at any moment for progressive or time-constrained rendering

Previous work and their limitations

- Adaptive sampling based on the -atistical nature of the estimation [Purgathofer 1987]

Not a relative error: does not take into account dynamic reduction during tonemapping

- Adaptive sampling based on and entropy measures [ $\mathrm{Xu}$ et al 2007] Does not make the error uniform during rendering, thus less time-constrained rendering

Both approaches can lead to poor sampling due to low-samples error estimations which

underestimate the actual error

\section{Our approach}

- Use a relative error to avoid focusing all processing power on areas receiving more energy. These zones are not necessarly the most noticeable after tonemapping, e.g. undersampling in shadows leads to highly visible noise

- Alternate between uniform and adaptive sampling, to ensure that error estimations of all pixels improve during rendering

\section{Robust Adaptive Sampling for Monte-Carlo-based rendering}

Anthony Pajot, Loïc Barthe, Mathias Paulin

Relative error and robustness to outliers

For each pixel, relative error:
$\quad e_{r}(I)=\frac{V\left(x_{1}, \ldots, x_{n}\right)}{E[X]}$
Standard estimation:
$\quad e_{a}(I)=\frac{V\left(x_{1}, \ldots, x_{n}\right)}{<I>}$

\section{$\rightarrow$ underestimation}
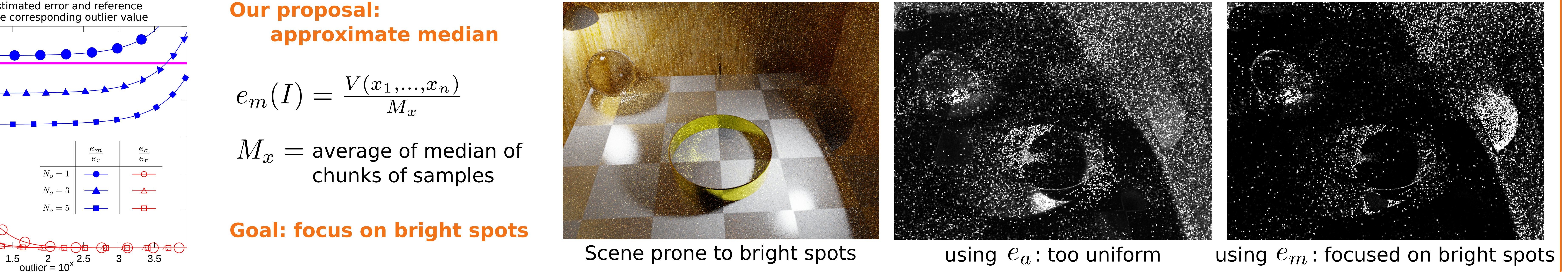

Alternation: avoid poor low-samples error estimation
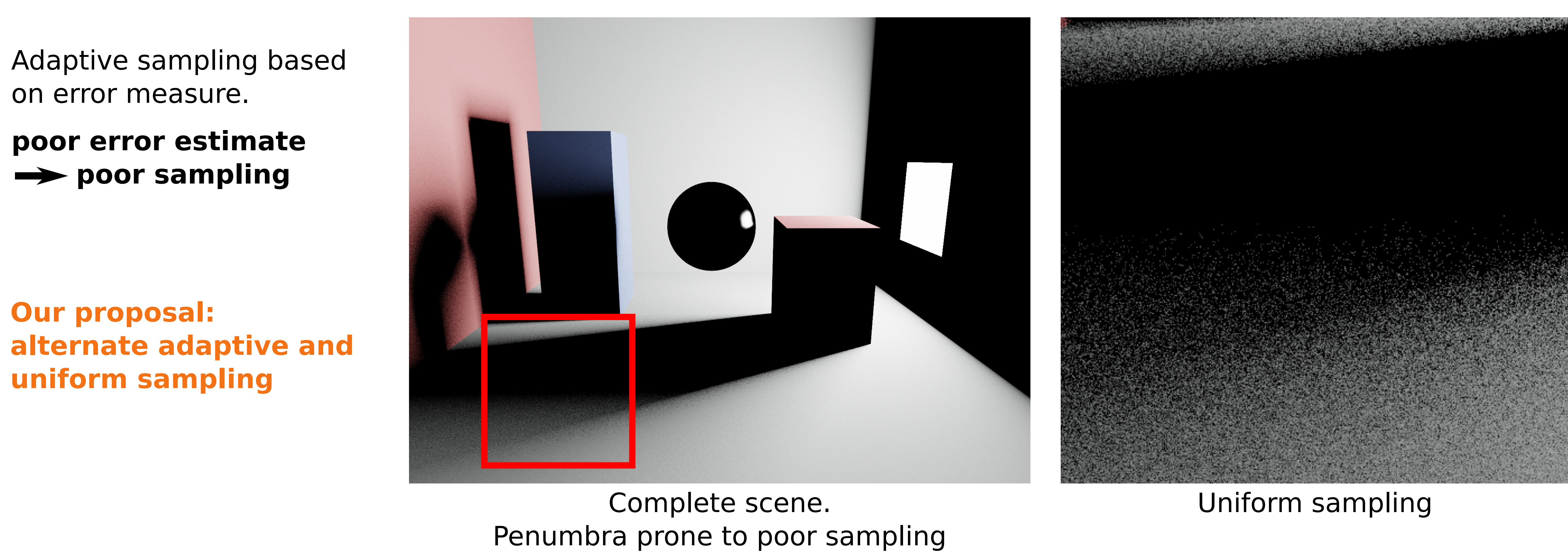

Uniform sampling

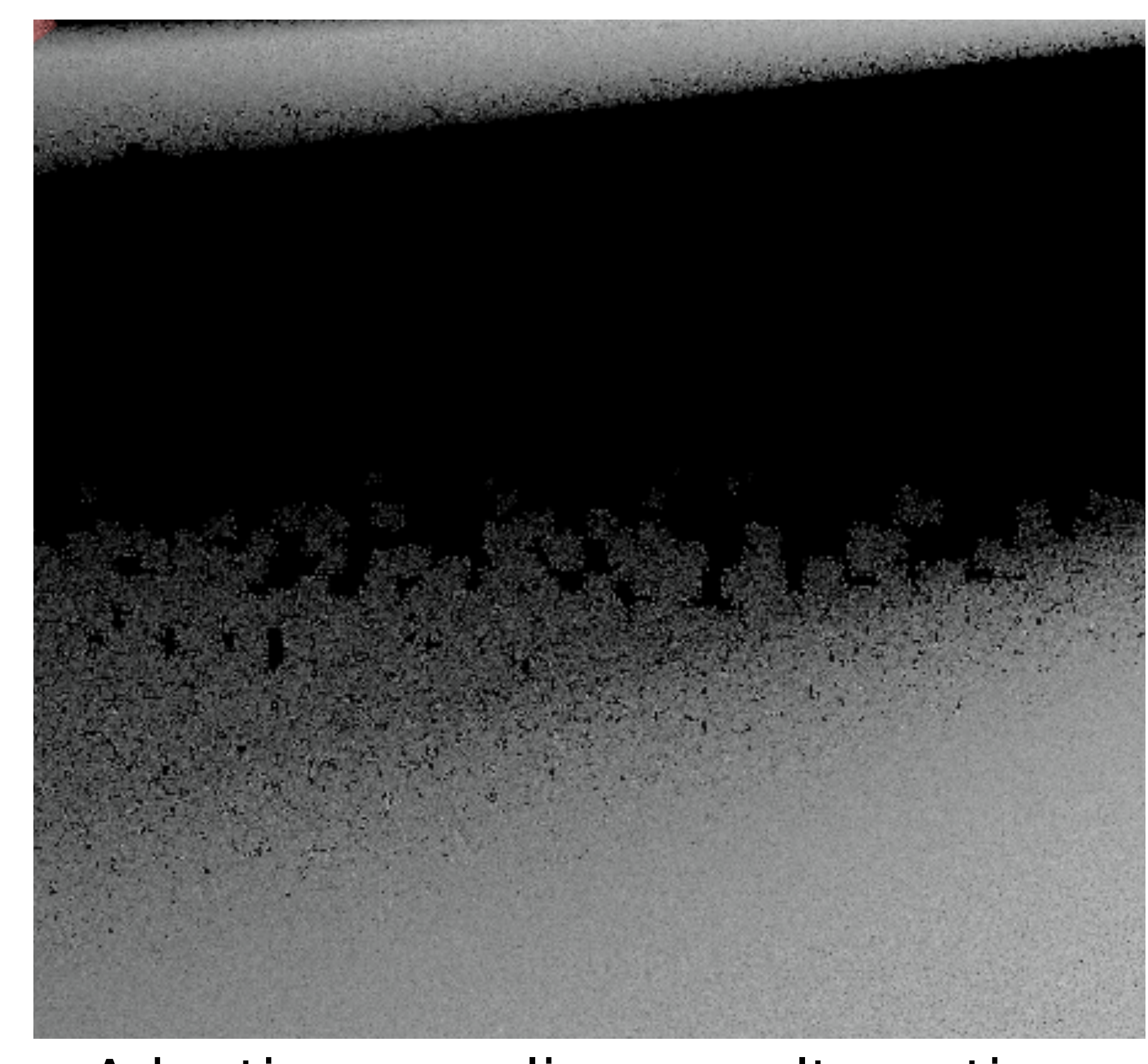

Patterns in th, no alternation Patterns in the penumbra

Adaptive sampling, alternation No patterns in the penumbra

Complete adaptive sampling algorithm

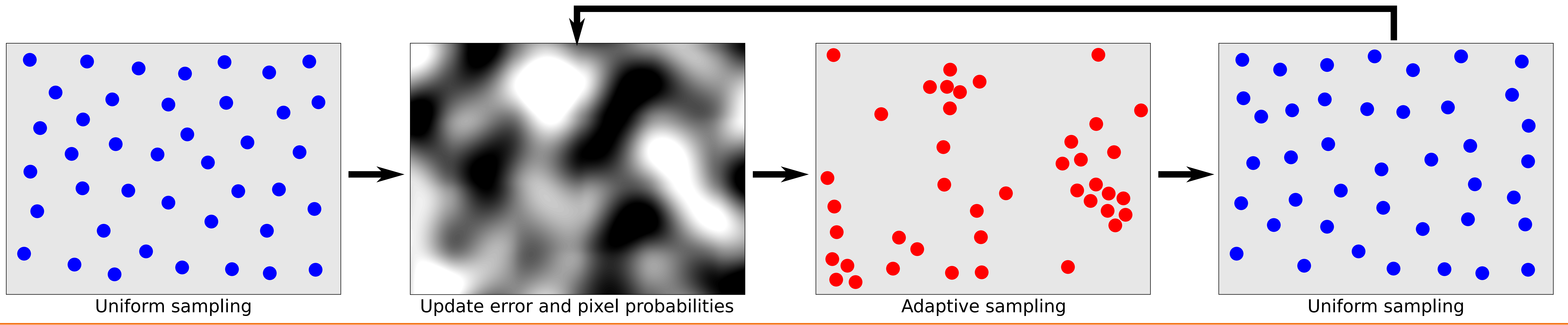

Comparison with Tsallis entropy [Xu, Sbert, Xinh and Zhan 2007]
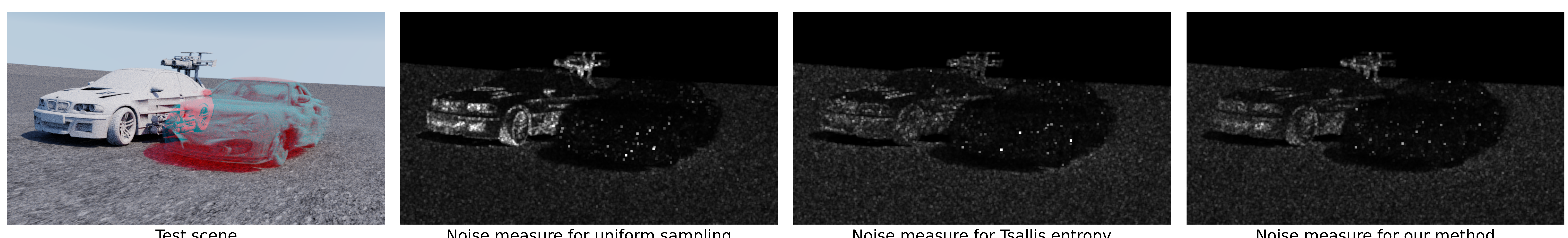

Noise measure for uniform sampling

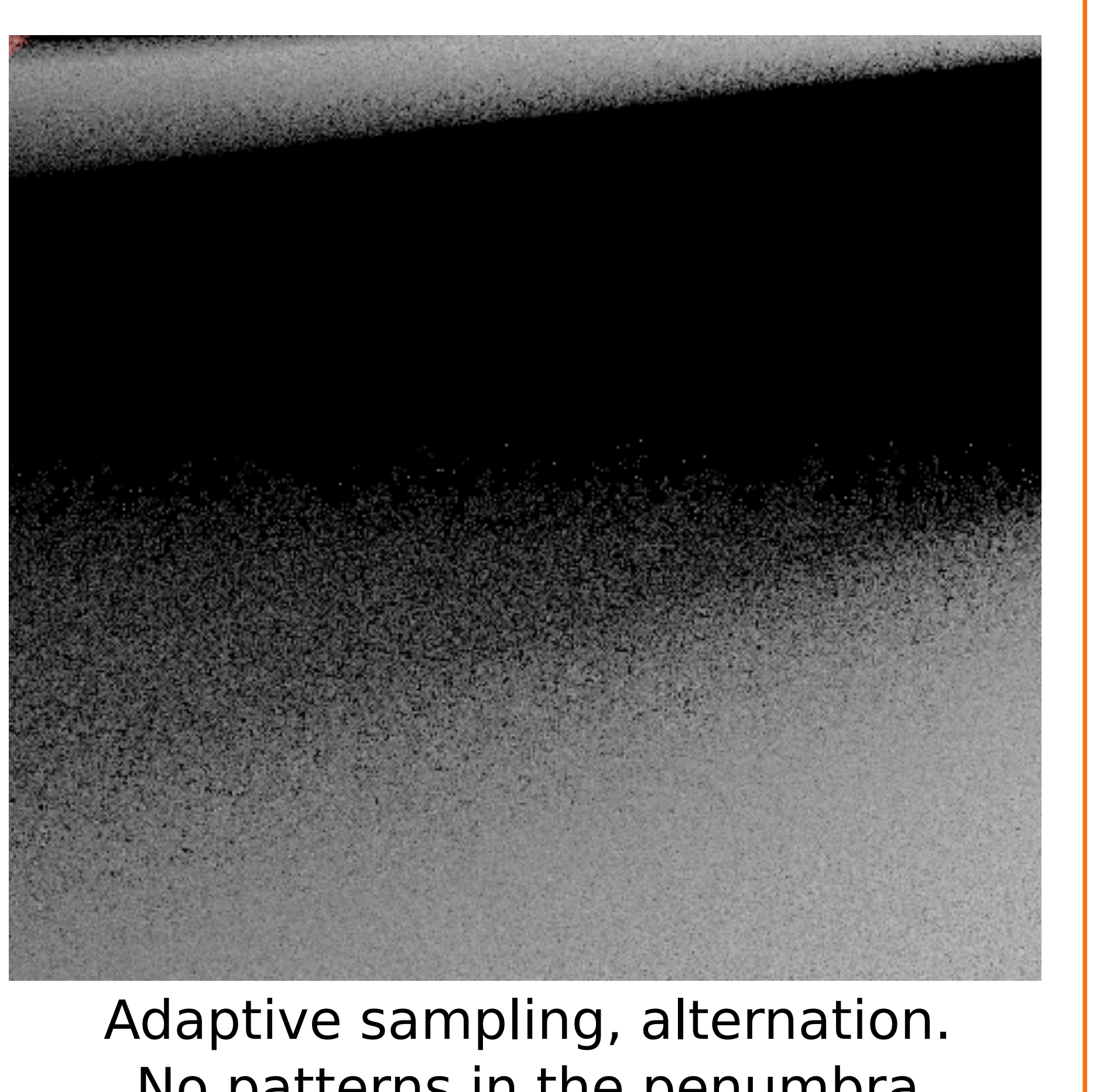

3 